Draft version November 11, 2018

Preprint typeset using $\mathrm{LAT}_{\mathrm{E}} \mathrm{X}$ style emulateapj v. 08/22/09

\title{
RATES AND DELAY TIMES OF TYPE IA SUPERNOVAE
}

\author{
Ashley J. Ruiter ${ }^{1,2}$, Krzysztof Belczynski ${ }^{3,4}$, Chris Fryer ${ }^{3}$ \\ ${ }^{1}$ New Mexico State University, Dept of Astronomy, 1320 Frenger Mall, Las Cruces, NM 88003 \\ 2 Harvard-Smithsonian Center for Astrophysics, 60 Garden St., Cambridge, MA 02138 (Predoctoral Fellow) \\ ${ }^{3}$ Los Alamos National Laboratory, Los Alamos, NM 87545 \\ 4 Oppenheimer Fellow \\ aruiter@nmsu.edu, kbelczyn@nmsu.edu, clfreyer@lanl.gov \\ Draft version November 11, 2018
}

\begin{abstract}
We analyze the evolution of binary stars to calculate synthetic rates and delay times of the most promising Type Ia Supernovae progenitors. We present and discuss evolutionary scenarios in which a white dwarf reaches the Chandrasekhar-mass and potentially explodes in a Type Ia supernova. We consider: Double Degenerate (DDS; merger of two white dwarfs), Single Degenerate (SDS; white dwarf accreting from H-rich companion) and AM Canum Venaticorum (AM CVn; white dwarf accreting from He-rich companion) scenarios. The results are presented for two different star formation histories; burst (elliptical-like galaxies) and continuous (spiral-like galaxies). It is found that delay times for the DDS in our standard model (with common envelope efficiency $\alpha_{\mathrm{CE}}=1$ ) follow a power-law distribution. For the SDS we note a wide range of delay times, while AM CVn progenitors produce a short burst of SNe Ia at early times. The DDS median delay time falls between $\sim 0.5-1$ Gyr; the SDS between $\sim 2-3 \mathrm{Gyr}$; and the AM CVn between $\sim 0.8-0.6 \mathrm{Gyr}$ depending on the assumed $\alpha_{\mathrm{CE}}$. For a Milky Way-like galaxy we estimate the rates of SNe Ia arising from different progenitors as: $\sim 10^{-4}$ $\mathrm{yr}^{-1}$ for the SDS and AM CVn, and $\sim 10^{-3} \mathrm{yr}^{-1}$ for the DDS. We point out that only the rates for two merging carbon-oxygen white dwarfs, the only systems found in the DDS, are consistent with the observed rates for typical Milky Way-like spirals. We also note that DDS progenitors are the dominant population in elliptical galaxies. The fact that the delay time distribution for the DDS follows a powerlaw implies more Type Ia supernovae (per unit mass) in young rather than in aged populations. Our results do not exclude other scenarios, but strongly indicate that the DDS is the dominant channel generating SNe Ia in spiral galaxies, at least in the framework of our adopted evolutionary models. Since it is believed that white dwarf mergers cannot produce a thermonuclear explosion given the current understanding of accreting white dwarfs, either the evolutionary calculations along with accretion physics are incorrect, or the explosion calculations are inaccurate and need to be revisited.

Subject headings: binaries: close — supernovae: general
\end{abstract}

\section{INTRODUCTION}

Type Ia Supernovae (SNe Ia) play an important role in astrophysics as cosmological distance indicators. Additionally, they provide iron peak elements, having direct consequences for the chemical evolution of galaxies (Riess et al. 1995; Matteucci \& Greggio 1986, de Donder \& Vanbeveren 2003). Currently $\gtrsim 2000$ SNe la have been observationally confirmed $^{1}$, some as distant as $z \sim 1.55$ (Strolger et al. 2004). Empirically-derived relationships between light curve properties and intrinsic luminosity (i.e., $\Delta m_{15}$ (Phillips 1993) and stretch-factor $s$, (Perlmutter et al. 1997)) have made it possible to 'standardize' absolute magnitudes of $\mathrm{SNe}$ Ia light curves over a wide variety of host galaxy environments. ${ }^{2}$ Their use as 'standard candles' on cosmological scales has led to the realization that the expansion rate of the universe is accelerating, and has enabled accurate estimations of $\Omega_{\Lambda}$ and $\Omega_{M}$ (e.g., Schmidt et al. 1998; Riess et al. 1998, Perlmutter et al. 1999). However, using SNe la in order to set the distance scale for the determination of cosmolog-

\footnotetext{
${ }^{1}$ http://www.cfa.harvard.edu/iau/lists/Supernovae.html

${ }^{2}$ It has been shown that SNe Ia originating among young stellar populations are overall more luminous than those associated with older stellar populations (i.e., Hamuy et al. 1995).
}

ical quantities requires the (still unfounded) assumption that the physical properties of their progenitors are unchanging with redshift.

Despite the continued use of SNe Ia as standard candle distance indicators, their origin remains uncertain. It is generally accepted that SNe Ia arise from the total disruption of a Chandrasekhar mass $\left(\sim 1.4 \mathrm{M}_{\odot}\right)$ carbonoxygen white dwarf (WD) as a result of thermonuclear explosion. This hypothesis is supported by the fact that the amount of energy observed in the explosions $\left(\sim 10^{51}\right.$ erg; Thielemann et al. (2004)) is equal to the amount which would be produced in the conversion of carbon and oxygen into iron (see e.g., Livio 2000, for a review). It is natural to presume that the exploding white dwarf must accrete matter from a close stellar companion until reaching the critical Chandrasekhar mass, though the nature of the companion, the rate and efficiency at which mass is accumulated onto the white dwarf, and which array of conditions are necessary in order for the white dwarf to ignite explosively, are not well understood (Nomoto et al. 1997).

In order to constrain the nature of SN Ia progenitors, the SN Ia rate has been studied as a function of parent galaxy stellar mass, star formation rate, colour, morphology, and radio power by several groups (e.g., Mannucci et 
al. 2005; Sullivan et al. 2006, Calura \& Matteucci 2006 Della Valle \& Panagia 2003). Mannucci et al. (2005) found that the SN la rate is higher in bluer, later type galaxies, supporting the hypothesis that there is a nonnegligible number of SNe Ia originating from young progenitors. Sullivan et al. (2006) found that the SN Ia rate per unit mass increases as a function of star formation activity, a trend which coincides with the results of Calura \& Matteucci (2006), who used chemical evolution models to derive the rate of SNe Ia as a function of galaxy Hubble type. Della Valle \& Panagia (2003) discovered an enhanced SN Ia rate in radio-loud galaxies compared to their radio quiet counterparts, possibly as a result of past interactions/mergers with dwarf galaxy companions leading to an increased number of newly-formed or captured young stars (see also Della Valle et al. (2005)). The evolution of the supernova rate (both core-collapse and Type Ia) as a function of cosmic time was investigated by Madau et al. (1998), who convolved a set of theoretical characteristic SN delay times with the cosmic star formation history, resulting in an estimate of SN rates out to intermediate redshifts. Later studies showed that a single-component delay time could not be reconciled with the observed mass of iron in galaxy clusters and the corresponding ratio of core-collapse to Ia SN rates (Maoz \& Gal-Yam 2004).

SNe la are observed in both young and old galaxies (Branch \& van den Bergh 1993), thus it is natural to presume that the progenitors may originate from both young and old stellar populations. Recently, it has been found that SNe Ia appear to span a wide range of delay times which is bimodal in nature, consisting of a 'prompt' population with short delay times, and a 'tardy' population whose average delay time distribution (DTD) is much wider and is best described by a decaying exponential function (Scannapieco \& Bildsten 2005: Mannucci et al. 2006 Dilday et al. 2008). However, whether the apparent bimodal DTD shape is limited to low-redshift SNe alone or whether it also applies to $\mathrm{SNe}$ at higher redshift is still unclear (Dahlen et al. 2008).

Two formation scenarios have emerged as the most likely channels for SNe Ia progenitors: The Single Degenerate Scenario (SDS, Whelan \& Iben 1973, Nomoto 1982 ) and the Double Degenerate Scenario (DDS, Iben \& Tutukov 1984; Webbink 1984). The SDS is encountered when a WD accretes H-rich matter during stable Roche Lobe Overflow (RLOF) from a stellar companion; either a main sequence or an evolved (giant) star. The WD increases in mass up to the Chandrasekhar mass limit, enabling carbon to ignite explosively in the WD center causing a SN Ia. The DDS is the result of a merger of two white dwarfs. If the combined mass of the merger exceeds the Chandrasekhar mass, the result may be a SN Ia. Additionally it has been suggested that a third channel, the AM CVn channel ${ }^{3}$, may account for $1 \%$ of SNe Ia (Solheim \& Yungelson 2005). AM CVn binaries (see e.g., Nelemans 2005: Warner 1995) are ultra-compact systems involving a WD accretor and a helium-rich donor exchanging matter via RLOF. The donors are expected to be small stars given the small orbital size (close orbits; $\lesssim 1 \mathrm{hr}$ ). Other possible SNe Ia formation scenarios have been proposed, though they likely do not account

\footnotetext{
${ }^{3}$ SWB-like in Belczynski et al. (2005).
}

for the majority of SNe Ia (e.g., sub-Chandrasekhar mass SNe Ia (Woosley \& Weaver 1994), common envelope WD mergers (Livio \& Riess 2003 ; Applegate 1991; Sparks \& Stecher 1974)).

In this study we follow the evolution of stellar populations in two different environments, that which is typical for an elliptical galaxy (instantaneous burst of star formation at $t=0$ ) and that typical for a spiral galaxy (continuous star formation). We also employ two different parameterizations for common envelope (CE) evolution. To substantiate our conclusions, we use exactly the same total stellar mass and metallicity for each population; the only differences in our model galaxies are the star formation histories, and the assumed CE removal efficiency $\alpha_{\mathrm{CE}}$. We show which SN Ia progenitors are the most likely (from an evolutionary perspective) for each host galaxy type, and derive delay times and rates for the most promising SNe Ia progenitor scenarios. We compare our results to those of previous studies and we discuss our results (e.g., in terms of explosion physics) in the last two sections, respectively.

\section{MODEL DESCRIPTION}

Our stellar evolution calculations are performed using the StarTrack population synthesis code. A detailed description of the input physics is presented in Belczynski et al. (2002, 2008). Single star evolution is followed from the ZAMS until remnant formation employing modified analytic formulae and evolutionary tracks from Hurley et al. (2000). Evolution of binary stars is more complex, and several processes important for field binary evolution are accounted for, such as tidal interactions, mass transfer phases, common envelope evolution, supernova kicks, magnetic braking and gravitational radiation (see Belczynski et al. 2008, for formulae). We incorporate recent prescriptions for mass growth of WDs, employing accretor mass-dependent accumulation efficiencies which may lead to nova explosions, stable burning, or optically thick WD winds (Nomoto et al. 2007, Kato \& Hachisu 2004; Hachisu et al. 1999| Kato \& Hachisu 1999| Prialnik \& Kovetz 1995 Hashimoto et al. 1986, see below). The physical properties of the stars are computed throughout the evolution.

A merger between two white dwarfs may lead to a DDS SN Ia. A detached WD-WD binary will eventually reach contact due to angular momentum loss from the emission of gravitational radiation, and if the binary configuration (e.g., mass ratio) leads to a merger, the less massive WD is accreted onto the more massive WD (see e.g., Tutukov \& Yungelson 1979). If the combined mass of the merger exceeds the Chandrasekhar mass, it is recorded as a potential DDS SN Ia progenitor. We assume a priori that every WD-WD merger with $M \geq 1.4 \mathrm{M}_{\odot}$ consisting of $\mathrm{CO}-\mathrm{CO}$, CO-He, or He-He WDs leads to an instantaneous SN Ia. ${ }^{4}$ Mergers involving other WD types occur in our simulations but we do not count them as SN Ia progenitors.

A SDS or AM CVn SN Ia may result from the accumulation of matter on a white dwarf's surface via stable RLOF from a stellar companion. For accretion of

\footnotetext{
${ }^{4}$ In Belczynski et al. (2005), it was assumed that a merger between any two WDs, including ONe WDs, with a combined mass exceeding $1.4 \mathrm{M}_{\odot}$ would lead to a SN Ia, though CO-CO mergers made up $88 \%$ of the DDS SNe Ia in that study.
} 
hydrogen-rich material, strong nova explosions inhibit the accumulation of hydrogen on the WD surface for very low mass transfer rates $<10^{-11} \mathrm{yr}^{-1}$ (Prialnik \& Kovetz 1995). For mass transfer rates above this threshold, we interpolate over the results of Prialnik \& Kovetz (1995) to obtain the mass accretion efficiencies, and we account for optically thick winds at high mass transfer rates (Hachisu et al. 1999, see also Belczynski, Bulik \& Ruiter (2005) Section 2.3 for a more detailed description). The only difference between the accumulation efficiencies in this work and those of Belczynski et al. (2005) is that here we have additionally included an updated prescription for accretion of hydrogen-rich matter from Nomoto et al. (2007), in which fully efficient accumulation is only achieved for a very narrow range of mass transfer rates, and is also dependent upon the white dwarf accretor mass (Nomoto et al. 2007, see equations $5 \& 6)$.

Accretion of helium-rich matter is treated in the same fashion as in Belczynski et al. (2005): accretion prescriptions are adopted from Kato \& Hachisu (1999). However this study contains one major difference which affects our results: though in this work we do allow for the formation of sub-Chandrasekhar mass $\mathrm{SNe} \mathrm{Ia}$, we do not include these binaries as potential SN Ia progenitors here. In Belczynski et al. (2005), a large fraction (61\%) of the Type Ia SNe which contributed to the presented delay times in the standard model were in fact sub-Chandrasekhar $\mathrm{SNe}$ Ia, in which the accumulation of $\sim 0.1 \mathrm{M}_{\odot}$ of He-rich material on the WD surface could lead to an edge-lit detonation and subsequent SN Ia (Woosley \& Weaver 1994 Kato \& Hachisu 1999).

The criteria used here for defining SNe Ia progenitors arising from different formation channels are different from the work of Belczynski et al. (2005). In this work, we only consider accreting WDs which have obtained a mass of $1.4 \mathrm{M}_{\odot}$ as potential SNe Ia. We make the distinction between SN Ia progenitors with $\mathrm{CO}$ white dwarfs accreting from non-degenerate hydrogen-rich companions (SDS), and white dwarfs accreting from helium-rich companions (AM CVn). We note that He WDs never reach the Chandrasekhar mass in our simulations with the adopted accumulation physics, and we have assumed that oxygen-neon-magnesium WDs collapse to form a neutron star (accretion induced collapse) upon reaching the Chandrasekhar mass, rather than producing a SN Ia (e.g., Miyaji et al. 1980). For the SDS and AM CVn cases, we record the binary as a SN Ia once the accreting WD has reached the Chandrasekhar mass. The SDS may occur when a WD accretes matter via RLOF from any hydrogen-rich companion (e.g., main sequence or evolved star). If the WD accumulates enough hydrogen on its surface such that steady burning can occur, the WD can increase in mass up to $1.4 \mathrm{M}_{\odot}$, carbon ignites explosively in the WD centre and the result is a SN Ia. In the AM CVn scenario, we assume the result is a $\mathrm{SN}$ Ia if the $\mathrm{CO} W D$ reaches $1.4 \mathrm{M}_{\odot}$ via stable $\mathrm{RLOF}$ from a helium-rich companion. We allow for different types of helium-rich donors in the AM CVn scenario: helium stars (non-degenerate stars burning helium in the core or in a shell which have been stripped of their outer hydrogen envelope), helium white dwarfs, and hybrid white dwarfs (CO-rich core, helium-rich mantle).

We adopt two contrasting star formation rates (SFR): an instantaneous burst at $t=0$ (elliptical galaxy) and a constant SFR for 10 Gyr (spiral galaxy). Both populations are then evolved up to $15 \mathrm{Gyr}$. The mass formed in stars in both cases is the same: $6 \times 10^{10} \mathrm{M}_{\odot}$, which corresponds to the stellar mass in the Milky Way (MW; Klypin et al. 2002). In each population we adopt a binary fraction of $50 \%$ (2/3 stars in binaries), though this fraction may be overestimating the binary population among low-mass stars (Lada 2006), and underestimating the binary fraction among massive stars (Kobulnicky \& Fryer 2007). All stars are evolved with solar-like metallicity $(Z=0.02)$. While the initial distributions representative of the physical characteristics of ZAMS binaries, and the correct way in which to treat common envelope evolution and magnetic braking are all somewhat uncertain, our choices for various distribution functions are constrained by available observations (see below). The magnetic braking prescription which we adopt is that of Ivanova \& Taam (2003), which is based on a twocomponent coronal model see Section 3.2 of Belczynski et al. 2008).

ZAMS masses (M $\left.M_{\text {ZAMS }}\right)$ span the entire mass range: $0.08-150 \mathrm{M}_{\odot}$. Single stars and binary primaries are drawn from a 3-component broken power law initial mass function (Kroupa et al. 1993), and secondary masses are obtained from a flat mass ratio distribution (Mazeh et al. (1992), $q=$ secondary/primary), which is the canonical choice among population synthesis studies (Lü et al. 2006). However, given the observational selection effects, the true mass ratio distribution among ZAMS binaries remains unknown, though it is likely dependent upon stellar mass (Trimble 1990, Duquennoy \& Mayor 1991). It has been suggested that the mass ratio distribution among local spectroscopic field binaries as well as young early-type stars is peaked near unity (Fisher et al. 2005: Kobulnicky \& Fryer 2007). Initial orbital separations in our calculations span a wide range up to $10^{5} \mathrm{R}_{\odot}$ and are drawn from a distribution $\propto 1 / a(\mathrm{Abt} 1983)$, which has been found to be representative of the local population of Hipparcos binaries (Lépine \& Bongiorno 2007). Initial eccentricities are drawn from a distribution $\Psi(e)=2 e$ (Duquennoy \& Mayor 1991).

Close binaries (and potential SN Ia progenitors) are believed to encounter a phase of common envelope evolution, and this remains one of the most poorly-understood phases in stellar astrophysics. For this reason, we present the delay times and rates of SNe Ia for two different CE parameterizations. Some comparison between different prescriptions for $\mathrm{CE}$ evolution have been tested against observations for local double white dwarfs (Nelemans \& Tout 2005). It is unclear at this point how the common envelope phase should best be treated in population synthesis studies, and currently detailed models are not sophisticated enough to explore the parameter space in detail (Ricker \& Taam 2008). For this work, we choose the ' $\alpha$ ' prescription for CE evolution (Webbink 1984), in which the orbital energy of the binary is used to unbind the common envelope from the system. We choose two different values for the parameterization of common envelope removal efficiency $\alpha_{\mathrm{CE}}$. For our standard model (Model 1) we choose $\alpha_{\mathrm{CE}} \times \lambda=1$, where $\lambda$ is a function of the donor envelope structure, and is of order unity (see also van der Sluys et al. 2006). As an alternative, we 
additionally include a model with decreased common envelope removal efficiency in which $\alpha_{\mathrm{CE}} \times \lambda=0.5$ (Model 2).

\section{DELAY TIMES}

Model 1: $\alpha_{\mathrm{CE}} \times \lambda=1$. We use the elliptical model, with all stars born at $t=0$ to demonstrate the delay time distribution for the various progenitors. Figure 1 (top panel) shows the characteristic delay times for Model 1, and the average and median delay times of all three progenitors are also indicated. The sharp cut-off near 15 Gyr is artificial, as evolution was only allowed to proceed for 15 Gyr.

The DDS events follow a power-law like DTD, with a median of $t_{\text {Med }}=0.93 \mathrm{Gyr}$ and approximated functional form of $f(t) \propto 100 t^{-1}$. Only a small fraction $\sim 5 \%$ are 'prompt' (Mannucci et al. 2006) events with delay times $t<100$ Myr. The DDS events are expected to be found long after the star formation has ceased $(t \sim 10-15 \mathrm{Gyr})$ therefore we expect these progenitors to produce SNe Ia both in young (spiral and starburst) and old (elliptical and bulges) host populations. Even though we have allowed DDS progenitors to arise from mergers between any combination of $\mathrm{CO}$ and/or He WDs, all of our DDS systems originate from mergers of CO-CO WD binaries, since mergers of He-He or CO-He WD binaries never exceed $1.4 \mathrm{M}_{\odot}$. The evolution leading to the formation of a CO-CO WD binary usually starts with two intermediatemass stars $\left(\mathrm{M}_{\mathrm{ZAMS}} \sim 3-9 \mathrm{M}_{\odot}\right)$ that evolve through a series of close interactions, the first one typically being stable RLOF and the last one being a CE phase. Once a CO-CO WD is formed, the dominant mechanism for angular momentum loss is gravitational radiation, the merger timescale $\tau_{G R} \propto a^{4}$ (Peters 1964). Given the initial distribution of orbital separations $\propto a^{-1}$, and the evolutionary orbital change that is to first order the same for all DDS progenitors (reduction of orbital size by a factor of $\sim 10-100$ during the CE phase), the delay time $t$ should likely then follow from the product of the initial distribution and the change in separation which occurs due to gravitational radiation:

$a^{-1} d a / d t \propto\left(t^{1 / 4}\right)^{-1} t^{-3 / 4} \propto t^{-1}$. Thus, it is not surprising that the DTD follows a power law as presented in Figure 1.

It is found that relatively speaking, the shortest delay times originate from the AM CVn channel. The distribution is somewhat narrow, with the majority of events occurring with $t<2 \mathrm{Gyr}$, with a median of $t_{\mathrm{Med}}=0.59$ Gyr. Most AM CVn progenitors originate from intermediate-mass stars (primaries $\mathrm{M}_{\mathrm{ZAMS}} \sim 5-7$ $\mathrm{M}_{\odot}$, secondaries $\left.\mathrm{M}_{\mathrm{ZAMS}} \sim 2-4 \mathrm{M}_{\odot}\right)$, however, in contrast to DDS progenitors, they undergo two CE events. After two CE phases the orbits of pre-AM CVn binaries are very close, which allows for rapid orbital decay due to emission of GR, and the final RLOF starts with no significant delay. At the onset of the final RLOF (start of AM CVn phase), the accretor is a massive CO WD and the companion is either a helium star $(65 \%)$ or a helium/hybrid WD (35\%). Once the RLOF commences, it proceeds on a short-timescale with high mass transfer rates $\left(\sim 10^{-5}-10^{-6} \mathrm{M}_{\odot} \mathrm{yr}^{-1}\right)$, enabling the $\mathrm{CO}$ WD to rapidly build up to the Chandrasekhar mass and subsequently produce a SN Ia explosion. Note that the potential SN Ia progenitor systems discussed here in the framework of the AM CVn scenario are by no means a representation of the observed sample of $\sim 22$ AM CVns. The rare systems discussed here are more massive, ultracompact systems (median $P_{\text {orb }} \sim 10 \mathrm{~min}$ ) and thus have higher mass transfer rates than the population of AM CVns which is presently observed (median $P_{\text {orb }} \sim 35$ min). The typical, low-mass AM CVn binaries are longlived systems which do not disappear from the observational population due to SN Ia disruptions. There is a clear observational bias against those AM CVn binaries which can potentially produce a SN Ia.

The SDS channel displays a rather flat (though somewhat decreasing) DTD over a wide range $t \sim 1-15$ Gyr, with a median of $t_{\text {Med }}=3.23 \mathrm{Gyr}$. The SDS systems are found to be binaries with a CO WD accreting from an evolved star ( 95\%; mostly red giants) or a main sequence star $(\sim 5 \%)$. Since the evolution leading to the formation of a CO WD is rather fast, the delay time for the SDS is set by the evolutionary timescale of the evolved donor. That in turn is a strong function of initial ZAMS donor mass $\left(0.7<\mathrm{M}_{\mathrm{ZAMS}}<2.7\right.$ $\mathrm{M}_{\odot}$; lifetimes $\left.0.5-30 \mathrm{Gyr}\right)$. The longest delay times follow from progenitors whose donors, on average, had the smallest initial masses. Mass transfer rates can be initially high; up to $\sim 10^{-3} \mathrm{M}_{\odot} \mathrm{yr}^{-1}$, in the case of giants with $M_{\mathrm{ZAMS}}>1 \mathrm{M}_{\odot}$, though hydrogen accumulation is only fully efficient for a narrow range of mass transfer rates (see Nomoto et al. 2007, Figure 4). Despite the high mass transfer rate from the donor, only a fraction of the hydrogen accumulates on the white dwarf (accumulation rates of $\sim 10^{-7} \mathrm{M}_{\odot} \mathrm{yr}^{-1}$ ). For SDS progenitors with main sequence donors, the two stars are brought into contact due to loss of orbital angular momentum from magnetic braking (convective secondary) and to a lesser extent, gravitational wave emission. Mass transfer and accretion rates are much lower $\left(\sim 10^{-11} \mathrm{M}_{\odot} \mathrm{yr}^{-1}\right)$, and the WD takes $\gtrsim 1$ Gyr to accrete up to the Chandrasekhar mass.

Model 2: $\alpha_{\mathrm{CE}} \times \lambda=0.5$. The delay time distribution for Model 2 is shown in Figure 1 (bottom panel). The change in the treatment of the $\mathrm{CE}$ evolution leads to smaller orbital separations after the common envelope phase, which affects the subsequent binary evolution and thus leaves an imprint on the resulting DTD.

As with Model 1, Model 2 DDS progenitors involve only CO-CO WD binaries. However with the decreased CE efficiency, many potential DDS progenitors merge before a detached white dwarf binary has been produced, thus there is a lack of DDS progenitors and there is an increase in the number of merging WD + AGB core systems. The same power-law curve from the top panel of Figure 1 is shown for comparison. The power-law-like shape of the DDS is present in Model 2 for delay times $\lesssim 6$ Gyr with an additional pile-up of progenitors at short delay times $(t \lesssim 1 \mathrm{Gyr})$. The pile-up is due to the fact that in Model 2, DDS progenitor systems which survive the CE phase (e.g., binaries which do not merge in the common envelope) are on closer orbits upon emerging from the $\mathrm{CE}$, thus they merge with relatively shorter delay times $\left(\tau_{G R} \propto a^{4}\right)$. This 'shift' to earlier delay times in the DTD from Model 1 to Model 2 serves to build a relatively stronger peak at short delay times, while at the same time decreases the number of DDS progenitors 
with delay times $\gtrsim 1$ Gyr. This is the reason for the shorter median delay time in Model 2: $t_{\mathrm{Med}}=0.52$, vs. that of Model $1\left(t_{\mathrm{Med}}=0.93\right)$.

The SDS channel of Model 2 is more efficient than that of Model 1 since the WD and the stellar companion are brought on a closer orbit during the CE phase. For this reason, wider systems which would have evolved to become double white dwarfs (e.g., AM CVn double degenerates) in Model 1 evolve into binaries with WDs accreting from non-degenerate companions in Model 2. In Model 2, the contribution of main sequence donors in the SDS channel is slightly higher since after the common envelope and formation of a CO WD, in many cases the stars are close enough for the secondary to fill its Roche lobe before evolving off of main sequence. The number of SDS progenitors involving main sequence donors is doubled relative to Model 1 and now constitutes $10 \%$ of the total SDS population (90\% are giant or sub-giant companions). SDS progenitors originate from systems with donor masses $\sim 0.7<\mathrm{M}_{\mathrm{ZAMS}}<2.7 \mathrm{M}_{\odot}$, many of which are $\mathrm{M}_{\mathrm{ZAMS}}>1.25 \mathrm{M}_{\odot}$ thus have main sequence lifetimes shorter than $\sim 5$ Gyr. The increased number of SDS progenitors at earlier delay times leads to a decrease in the SDS median delay time from $\sim 3.2$ Gyr (Model 1) to $\sim 2.1$ (Model 2).

The DTD of the AM CVn progenitor population of Model 2 is notably different from that of Model 1, in that it is bimodal. The 'fast' channel at delay times $\lesssim 3$ Gyr is still present, and originates from progenitors with donor masses $\mathrm{M}_{\mathrm{ZAMS}} \gtrsim 2.5 \mathrm{M}_{\odot}$ which undergo two $\mathrm{CE}$ events. However in Model 2, the decreased orbital separation of post-CE binaries allows for the formation of a new SN Ia progenitor channel, in which a CO WD initially accretes hydrogen from a low-mass main sequence star for several Gyr, as the main sequence star continues to fuse hydrogen into helium in its core (see also Podsiadlowski 2008). The main sequence star, which has been losing its outer layers in RLOF, has built up a significant helium core by the time its mass is depleted to the hydrogen burning mass limit $\left(0.08 \mathrm{M}_{\odot}\right)$. Upon reaching the hydrogen-burning mass limit, the donor is said to be degenerate, and since it is helium-rich (exposed core) we treat it as a helium white dwarf. There is a brief period (several Myr) where RLOF ceases due to the decreased size of the newly degenerate helium WD before the stars are brought in contact again due to emission of gravitational radiation. Stable RLOF begins between the $\mathrm{CO}$ WD and helium WD, until the CO WD accretes up to the Chandrasekhar mass, exploding in a SN Ia. This additional (and scarcely-populated) 'slow' AM CVn channel with delay times $>6$ Gyr originates from progenitors with donor masses $0.7<\mathrm{M}_{\mathrm{ZAMS}}<1.0 \mathrm{M}_{\odot}$, which only undergo one $\mathrm{CE}$ event, and evolve through an active cataclysmic variable $(\mathrm{CV})$ phase for $\gtrsim$ a few Gyr. Under the Model $1 \mathrm{CE}$ evolution, such a system would be found as a detached COWD + MS binary, and would not make a $\mathrm{CV}$, nor a SN Ia, in a Hubble time.

\section{RATES}

Assuming a binary fraction of $50 \%$, as we have done in this study, $0.17 \%$ and $0.09 \%$ of stellar systems (where a 'system' represents either a single star or a binary) evolve into SNe Ia in Model 1 and Model 2, respectively. For an assumed binary fraction of $100 \%, 0.34 \%$ and
$0.18 \%$ of binaries evolve into SNe Ia in Model 1 and Model 2, respectively. Note that this applies to both elliptical and spiral populations as they differ only in SFR but not in any evolutionary parameters other than $\alpha_{\mathrm{CE}} \times \lambda$. The above fractions translate into integrated efficiencies of $1 \mathrm{SN}$ Ia per $2500 \mathrm{M}_{\odot}$ and $4700 \mathrm{M}_{\odot}$ of formed stars for Models 1 and 2, respectively, when a binary fraction of $50 \%$ is assumed. For an assumed binary fraction of $100 \%$ the integrated efficiencies are 1 SN Ia per $1500 \mathrm{M}_{\odot}$ and $1 \mathrm{SN}$ Ia per $2800 \mathrm{M}_{\odot}$ for Models 1 and 2, respectively. Though the efficiency scales with the adopted binary fraction, the rates as a function of time, obviously, are different depending on the adopted SFR.

A general summary of Model 1 and Model 2 rates is presented in Tables $1 \& 2$ where we show the rate of $\mathrm{SNe}$ Ia in $\mathrm{SNuM}$, where $1 \mathrm{SNuM} \equiv 1 \mathrm{SN}(100 \mathrm{yr})^{-1}\left(10^{10}\right.$ $\left.\mathrm{M}_{\odot}\right)^{-1}$ (Mannucci et al. 2005). We show the number of SNe la in SNuM for the three Chandrasekhar-mass models investigated in this work at 4 different epochs: $0.5,3,5$ and 10 Gyr. SNuM rates are shown for our four galaxy models: elliptical, Model 1; spiral, Model 1; elliptical, Model 2; spiral, Model 2.

Model 1. In Figure 2 (top panel), SN Ia rates (number of SNe per unit time) are shown for our Model 1 elliptical galaxy $\left(\alpha_{\mathrm{CE}} \times \lambda=1\right.$, instantaneous starburst at $\left.t=0\right)$. The three progenitor types: DDS, SDS and AM CVn are shown separately. The DDS rate declines with time, but DDS progenitors are found both at early and late times. The DDS events dominate over the other progenitor types. This dominance is very strong $(\sim 1-2$ orders of magnitude) and holds for the entire evolution of an elliptical galaxy $(0-15 \mathrm{Gyr})$. The rate of $\mathrm{SNe}$ Ia from $\mathrm{AM} \mathrm{CVn}$ systems is high for times $\lesssim 2 \mathrm{Gyr}$ and then rapidly declines. Since for a typical AM CVn progenitor (i) the CO WD forms early $\left(t_{\text {evol }}<100 \mathrm{Myr}\right)$ and with a high mass $\left(M_{\mathrm{COWD}} \sim 1.1 \mathrm{M}_{\odot}\right.$; from primaries of initial mass $M_{\text {ZAMS }} \sim 5-7 \mathrm{M} \odot$ ), and (ii) the orbital separation after two common envelopes is small ${ }^{5}$, the two stars are brought into contact either by GR (He WD) or a combination of GR and evolutionary expansion (helium star). Once the donor fills its Roche Lobe, accumulation is fully efficient for binaries with helium star donors (mass transfer rates $\sim 10^{-8} \mathrm{M}_{\odot} \mathrm{yr}^{-1}$ ), though in the case of helium WD donors, the mass transfer rate is initially higher $\left(10^{-5}-10^{-6} \mathrm{M}_{\odot} \mathrm{yr}^{-1}\right)$ due to the smaller stellar separation of these systems upon reaching contact $\left(\sim 0.3\right.$ vs. $\left.1.0 \mathrm{R}_{\odot}\right)$. For these relatively high mass transfer rates, only a fraction $(\sim 50 \%)$ of the mass is accreted by the CO WD. In either case, the mass accretion rate averaged over time from contact to $\mathrm{SN}$ Ia is found to be on the order of $10^{-7}-10^{-8} \mathrm{M}_{\odot} \mathrm{yr}^{-1}$ for AM CVn SN Ia progenitors, thus it takes $\sim 10-100 \mathrm{Myr}$ for the CO WD to accrete up to the Chandrasekhar mass. Therefore, the delay time is: $t_{\text {evol }}+(\sim 10-100)$ Myr $\left(+t_{\mathrm{GR}}\right.$ in the case of helium WD donors $)=0.2-2$ Gyr for a typical AM CVn SN Ia progenitor to produce a SN Ia.

The SDS rate maintains a nearly constant SN Ia rate through about $6-7 \mathrm{Gyr}$ and then drops by almost an order of magnitude. The increased rate at shorter de-

${ }^{5} a \sim 0.3-1 \mathrm{R}_{\odot}$, where the primary is a CO WD and the companion is either a helium star $\left(M_{\mathrm{He}} \sim 0.4 \mathrm{M}_{\odot}\right)$ or a helium WD $\left(M_{\mathrm{HeWD}} \sim 0.3 \mathrm{M}_{\odot}\right)$. 
lay times is due mainly to systems with evolved donors which encounter, on average, RLOF on shorter (evolutionary) timescales than their main sequence counterparts for which the RLOF is encountered on longer (magnetic braking) timescales.

The resulting rate of potential DDS SNe Ia varies substantially with time. At early times $(t \lesssim 1 \mathrm{Gyr})$ the rates are very high $\sim 0.01 \mathrm{yr}^{-1}$, and then they gradually decrease to reach $\sim 0.0003 \mathrm{yr}^{-1}$ at late times $(t \gtrsim 10$ Gyr). The observed rates for elliptical galaxies are estimated at the level of $\mathcal{R}_{\text {obs }} \sim 0.0018 \pm 0.0006 \mathrm{vr}^{-1}$ per unit $\left(10^{10} L_{\odot}^{B}\right)$ of blue luminosity (Cappellaro et al. 1999). As the blue luminosity of elliptical galaxies declines with time (after an early star formation episode), the rates presented in the top panel of Figure 2 should be corrected downwards at early times, while at later times they should be increased if our rates are to be compared with those of typical ellipticals. Obviously, the burst of star formation on the order of $6 \times 10^{10} \mathrm{M}_{\odot}$ would produce a blue luminosity larger than $10^{10} L_{\odot}^{B}$, while $10-15 \mathrm{Gyr}$ after the episode when stars more massive than $\sim 1 \mathrm{M}_{\odot}$ have formed remnants and are no longer contributing to the galaxy's light, the blue luminosity is smaller than the normalising value. Since we do not really know the distribution of age of the galaxies in the observed sample of ellipticals that were used in the SN Ia rate estimate, we do not attempt to correct our synthetic rates for the evolution of blue luminosity and we do not compare them directly to the observed rates of Cappellaro et al. (1999). However, we note that the observed rate is consistent with our predicted rates for the DDS progenitor, while the predicted rates for other progenitors (SDS and AM CVn) seem to be significantly too low.

In Figure 2 (bottom panel), we show the SN Ia rates for the spiral galaxy model of Model 1. It is found that DDS rates of SNe Ia at the current epoch are $0.002 \mathrm{yr}^{-1}$. At first, the DDS rate increases with time (after the onset of star formation), then remains approximately constant until the star formation stops leading to an overall decline in the rate. This behavior reflects the specific shape of the delay time distribution for the DDS combined with the SFR for our spiral galaxy model. The rates for SDS and AM CVn progenitors are much smaller and at the level of $\sim 10^{-4} \mathrm{yr}^{-1}$. SDS progenitors can generate $\mathrm{SNe}$ Ia long after star formation has ceased (long delay times), while AM CVn events disappear shortly after the star formation has stopped (short delay times). For comparison, over-plotted are empirical rates of SNe Ia. The rates were adopted from Cappellaro et al. (1999) for a Milky Way type spiral (Sbc-Sd) with a blue luminosity of $2 \times 10^{10} \mathrm{~L}_{\odot}$, and the rates are $\mathcal{R}_{\text {obs }}=0.004 \pm 0.002$ SN Ia $\mathrm{yr}^{-1}$. The DDS rate alone is consistent with the empirical rate of SNe Ia. The SDS and AM CVn SN Ia rates do not even come close to the empirical rate, and their addition to the DDS rate does not significantly affect the overall rates at any epoch. We note that our mass normalization which implies a constant star formation history for $10 \mathrm{Gyr}$ results in a SFR at the level of $6 \mathrm{M}_{\odot} \mathrm{yr}^{-1}$. The global SFR in the MW mav be somewhat lower: $\sim 3.5 \mathrm{M}_{\odot} \mathrm{yr}^{-1}$ (Cox 2000, O'Shaughnessy et al. 2008), and in that case the DDS rates are only marginally consistent with the observed Cappellaro et al. (1999) rates. On the other hand, it has been suggested that the SFR of the MW has been decreasing with time, only reaching $\sim 3.5 \mathrm{M}_{\odot} \mathrm{yr}^{-1}$ at the current epoch (Nelemans et al. 2001, 2004, see sect. 2.2). If such an estimate had been used the average SFR of the MW is found at the level of $\sim 8 \mathrm{M}_{\odot} \mathrm{yr}^{-1}$, and our results would scale up, being consistent with the DDS scenario as the major SN Ia contributor in MW-like spiral galaxies, as long as the Cappellaro et al. (1999) rates are being used for comparison.

Model 2. There is a marked decrease, by nearly a factor of 2, in the total number of SNe Ia progenitors in our model with decreased CE removal efficiency. The overall decrease is due to the fact that the most dominant potential channel, the DDS, is only $\sim 50 \%$ as efficient, since a larger fraction of binaries will merge in the common envelope phase rather than surviving the CE to subsequently form a double white dwarf.

In Figure 3 (top panel), SN Ia rates are shown for our Model 2 elliptical galaxy $\left(\alpha_{\mathrm{CE}} \times \lambda=0.5\right.$, instantaneous starburst at $t=0)$. DDS SNe Ia progenitors continue to outnumber the SDS and AM CVn progenitors, however there are some notable differences. For short delay times $t \sim 1 \mathrm{Gyr}$, the DDS rates are nearly a factor of 2 lower than they are for Model 1. Then at later times, the Model 2 DDS rates are a factor of $\sim 3$ below those of Model 1, reaching $\sim 9 \times 10^{-5} \mathrm{yr}^{-1}$ at delay times of $10 \mathrm{Gyr}$ (vs. $3 \times 10^{-4} \mathrm{yr}^{-1}$ for Model 1). Despite the lower DDS rates of Model 2, potential progenitors are found at all delay times, as they are in Model 1. The CE efficiency of Model 1 allows for DDS progenitors to be drawn from a wider range (going to smaller values) among the distribution of initial separations, where as progenitors with small initial separation in Model 2 are removed from the DDS population in mergers during the CE phase. The Model 2 SDS channel is more efficient (by a factor of 3 ) than the Model 1 SDS channel since post-CE WD + MS binaries are found on closer orbits. One major difference between the elliptical galaxy Ia rates of Model 1 and Model 2 is that the SDS rates match those of the DDS rates for delay times $\sim 2.5-5.5 \mathrm{Gyr}\left(\sim 0.0002 \mathrm{yr}^{-1}\right)$. Since the majority of the donors are evolved stars (giants or sub-giants), the delay involves two components: the main sequence lifetime of the donor (a few Gyr for a donor to become a giant) and the accretion timescale, over which the primary WD can increase its mass to the Chandrasekhar mass (10-100 Myr). Thus the main sequence lifetime of the donor is what sets the delay times for the SDS DTD. Since the stars are found on closer orbits after the common envelope, RLOF is encountered between the WD and the non-degenerate companion more often in Model 2 (typically when the donor is a sub-giant). Rates of potential AM CVn SN Ia are lower than those of Model 1 for elliptical galaxies and are at the level of $\sim 0.0002$ $\mathrm{yr}^{-1}$. For the majority of the progenitors the delay times are very short, so as in Model 1, these type of events are expected only in young host galaxies or in regions with ongoing star formation. 'Fast' AM CVn progenitors are more rare in Model 2 since these systems more readily merge in one of the two common envelope phases that lead to the formation of these progenitors. In contrast to Model 1, there is a small contribution of the 'slow' AM CVn progenitors (long delay times) in Model 2. The AM CVn channel is outnumbered by both the SDS and DDS 
channels at all epochs in the Model 2 elliptical galaxy.

In Figure 3 (bottom panel), SN Ia rates are shown for our Model 2 spiral galaxy. It is found that DDS rate of $\mathrm{SNe}$ Ia at the current epoch $(10 \mathrm{Gyr})$ is 0.001 $\mathrm{yr}^{-1}$; a factor of two below that of the Model 1 spiral galaxy. At first, the DDS rate increases and then remains fairly constant until star formation ceases at 10 Gyr. At all epochs (particularly during star formation), the DDS channel rates significantly outnumber the SDS and AM CVn channels, but to a lesser degree than when compared with Model 1. The SDS channel also exhibits a relatively constant rate at times later than $\sim 1$ Gyr. At the current epoch the SDS rates are at the level of 0.0002 $\mathrm{yr}^{-1}$; a factor of $\sim 5$ below the DDS rates. The rates arising from AM CVn progenitors are fairly negligible at all epochs $\left(<10^{-4} \mathrm{yr}^{-1}\right)$. Even when the rates from all three progenitor channels in the considered galaxy

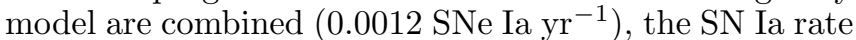
at the current epoch falls below the empirically-derived rate from Cappellaro et al. (1999) by roughly a factor of two.

It is worth comparing our model galaxy rates to the rates presented in Mannucci et al. (2005). In that study, Mannucci et al. (2005) derive SN rates for galaxies of various morphological types, and present the $\mathrm{SN}$ rates in SNuM (as inferred from $K$-band luminosity measurements). We cannot directly compare our rates in Tables $1 \& 2$ to those of Mannucci et al. (2005) since we do not know the exact ages of the galaxies in their sample. However, we note that our Model 1 elliptical galaxy SN Ia rate at $10 \mathrm{Gyr}^{6}$ is $0.005 \mathrm{SNuM}$ (see Table 1 DDS rates), which is nearly a factor of 10 below the SN Ia rate in E/S0 galaxies presented by Mannucci et al. (2005): $0.044_{-0.014}^{+0.016}$ SNuM (see their Table 2). We note as well that for the same model galaxy, at $t=500 \mathrm{Myr}$ (shortly after a burst of star formation at $t=0$ ) we obtain a SN Ia rate of $\sim 0.18$ SNuM (mostly via the DDS channel with some contribution from AM CVn), which is about a factor of 2 lower than the range of SN Ia rates found for star forming Irregular galaxies in Mannucci et al. (2005) $\left(0.77_{-0.31}^{+0.42}\right)$. The Mannucci et al. (2005) SN Ia rate for $\mathrm{S} 0 \mathrm{a} / \mathrm{b}$ spirals is found to be $0.065_{-0.025}^{+0.027} \mathrm{SNuM}$, which matches our Model 1 spiral galaxy rates at 5 Gyr $(0.065$ SNuM; mostly DDS \& AM CVn progenitors). The SN Ia rate of Sbc/d spirals from Mannucci et al. (2005) is $0.17_{-0.063}^{+0.068}$, and matches our Model 1 spiral SN Ia rate only at very early times $(\sim 0.5-1 \mathrm{Gyr}$, see Table 1$)$.

For the Model 2 elliptical galaxy, we find a low SN Ia rate of $\sim 0.001 \mathrm{SNuM}$ at $10 \mathrm{Gyr}$, which is over an order of magnitude below the rate for $\mathrm{E} / \mathrm{S} 0$ galaxies in Mannucci et al. (2005) $\left(0.044_{-0.014}^{+0.016} \mathrm{SNuM}\right)$. The rate at $500 \mathrm{Myr}$ for the same galaxy is $\sim 0.14 \mathrm{SNuM}$ (see Table 2); mostly arising from DDS progenitors with some contribution from AM CVn and SDS. This rate is a factor of a few below the Ia rates for Irregular galaxies in Mannucci et al. (2005) $\left(0.77_{-0.31}^{+0.42}\right)$. In comparing our Modei 2 spiral rates, we find that our SN Ia rate at $3 \mathrm{Gyr}$ $(\sim 0.07$ SNuM; mostly DDS progenitors with some contribution from SDS) is within the range of rates presented

6 A typical age for local ellipticals; see Mannucci et al. 2005 Section 7 . in Mannucci et al. (2005) for S0a/b spirals $\left(0.065_{-0.025}^{+0.027}\right)$, while only our spiral rates for Model 2 at very early times ( $<1$ Gyr; 0.13 SNuM, DDS) are high enough to match those of Mannucci et al. (2005) for Sbc/d type galaxies $\left(0.17_{-0.063}^{+0.068}\right)$. We note that in general, our rates (per unit mass) are lower than those of Mannucci et al. (2005), indicating that perhaps other channels leading to the formation of SNe Ia should be considered in evolutionary studies (e.g., single stars, sub-Chandrasekhar mass SNe Ia). We also note however that our predicted rates as a function of time (at least for the DDS) are consistent with the observed rates presented by Cappellaro et al. (1999).

We find that in general the DDS outnumber SDS and AM CVn progenitors. This effect is somewhat more pronounced in Model 1 (a factor of $\gtrsim 10$ ) than in Model 2 (a factor of $\sim 5$ ), but the reason why is clear for both models. The occurrence rate of a CO-CO WD merger with a total mass $\geq 1.4 \mathrm{M}_{\odot}$ (DDS) is higher than that of building up a CO WD's mass to $\sim 1.4 \mathrm{M}_{\odot}$ via stable mass transfer (SDS) in a binary. Formation efficiencies in both of the above cases are very low, after all SNe Ia are rather rare events. However, relatively speaking it is easier to find a pair of two CO WDs (DDS), each with a mass of $\gtrsim 0.7 \mathrm{M}_{\odot}$ which is a typical mass for CO WDs, than it is for a CO WD to double its mass through accretion (SDS/AM CVn). This finding is a consequence of the recent updates on accumulation physics calculations (Hachisu et al. 1999, Kato \& Hachisu 1999, Nomoto et al. 2007) that we have adopted in our evolutionary study. Basically, the accumulation onto a WD is hampered by a number of processes that tend to remove matter which is transferred from the companion in a close binary (e.g., nova explosions, He-shell flashes, optically thick winds from the surface of an accreting WD), in some cases leading to the disruption of an accreting WD before it reaches the limiting Chandrasekhar mass; i.e., accretion of $\sim 0.1 \mathrm{M}_{\odot}$ of a He-rich layer which ignites and disrupts the underlying WD (sub-Chandrasekhar mass SN Ia, see e.g., Kato \& Hachisu 1999). Since, (i) there is a rather narrow range of mass transfer rates which may lead to efficient accumulation and (ii) there are not that many binary configurations (and we have considered the entire range for our adopted evolutionary model) that can sustain mass transfer for a prolonged period of time, the SDS and AM CVn channels are found to produce SNe Ia at very low rates.

\section{COMPARISON WITH OTHER STUDIES}

The recent theoretical study of Hachisu et al. (2008) finds a SDS delay time distribution which follows a power law. In their study, Hachisu et al. (2008) incorporate a new mass stripping effect (based on Hachisu et al. (1999)), where in the case of high mass transfer rates the WD blows an optically thick wind strong enough to 'strip' material from a main sequence or giant donor. This effect in return stabilizes mass transfer, enabling the binary to avoid a CE phase even in the case of a relatively massive $\left(\sim 6 \mathrm{M}_{\odot}\right)$ donor. The result is that the WD can accrete stably up to the Chandrasekhar mass, with a wider range of potential progenitor donor ZAMS masses: $0.9-6 \mathrm{M}_{\odot}$ in Hachisu et al. (2008) vs. $0.7-2.7$ $\mathrm{M}_{\odot}$ in our current study. Even though we allow for SN Ia progenitors to form from any initial mass spanning 
the initial mass function, our SDS SNe Ia only derive from binaries involving low-mass donors since we do not take into account this stripping effect. We note that the Hachisu et al. (2008) model predicts the presence of a thick disc of hydrogen-rich circumstellar material around the SN Ia progenitor. If such a circumstellar torus were present around the majority of progenitors of SNe Ia, one would likely expect to observe hydrogen in their spectra, though to date less than $1 \%$ of SNe Ia have shown any signature of associated hydrogen (see e.g., Han \& Podsiadlowski 2006).

Han \& Podsiadlowski (2004) investigated SNe Ia progenitors from $\mathrm{WD}+\mathrm{MS}$ and $\mathrm{WD}+$ evolved binaries with very specific binary configurations using population synthesis. In that work, they do not present rates of $\mathrm{SNe}$ Ia derived from other possible formation channels of $\mathrm{SNe}$ Ia so we cannot compare DDS rates. The Galactic rate of SDS SNe Ia for their model which most closely matches our standard (Model 1) parameters is $\sim 6 \times 10^{-4} \mathrm{yr}^{-1}$. This value is an order of magnitude above our SDS spiral galaxy Model 1 rate of $\sim 6 \times 10^{-5} \mathrm{yr}^{-1}$, though is still nearly an order of magnitude lower than the empirical SN Ia Galactic rate of Cappellaro et al. $\left(1999,4 \times 10^{-3}\right.$ $\left.\mathrm{yr}^{-1}\right)$. The rate for the Han \& Podsiadlowski (2004) model which most closely matches Model 2 is $\sim 10^{-3} \mathrm{SNe}$ Ia $\mathrm{yr}^{-1}$, which is close (though still below) the Galactic rates of Cappellaro et al. (1999), and is a factor of 5 times higher than our Model 2 SDS rates $\left(\sim 2 \times 10^{-4} \mathrm{yr}^{-1}\right)$.

It was pointed out by Han \& Podsiadlowski (2004) that their prescription for hydrogen accumulation is more efficient than that used by other authors (i.e., Yungelson \& Livio 1998). It is also more efficient than the prescription we have adopted in this study. The range of hydrogen accretion rates onto WDs which leads to stable burning (see section 2) and efficient mass accumulation is uncertain, and it is possible that stable hydrogen burning may occur for a wider range of accretion rates, in turn allowing for higher SNe Ia rates following from the SDS channel as allowed in Han \& Podsiadlowski (2004). However, comparison of model hydrogen-accreting WDs on the H$\mathrm{R}$ diagram with supersoft X-ray sources (Nomoto et al. 2007 ) indicates that the prescription for stable hydrogen burning in a thin shell (and adopted here) is consistent with observations.

It is worth noting that the delay time distributions of Greggio (2005), derived using analytical formulations, produce a DDS DTD shape which is similar to ours: peaked at short $(<1 \mathrm{Gyr})$ delay times, followed by a smooth drop-off as a function of time, due to the dependence of the delay time on the timescale associated with gravitational wave emission. A similar trend is also found for the delay times of DDS progenitors in Yungelson \& Livio (2000, Fig. 2). The Greggio (2005) study also determined that the shape of the DTD arising from SDS progenitors was more flat than when compared to that of the DDS, and that the SDS delay time depended upon the main sequence lifetime of the secondary star (see their section 5), which is consistent with our findings.

Delay times of SNe Ia were calculated by Belczynski et al. (2005). It was found that the merger of two white dwarfs was consistent with an empirical delay time esti- mate of $\sim 3$ Gyr $(\text { Strolger et al. 2004 })^{7}$, and that WDs accreting from non-degenerate stars could potentially explain the observed delay times if a low common envelope efficiency is used $\left(\alpha_{\mathrm{CE}} \times \lambda=0.3\right)$ and if it is presumed a priori that WD mergers contribute negligibly or not at all to the SNe Ia population. The above results were obtained with an earlier version of the StarTrack code; the code was recently updated to include the most recent accumulation rates (e.g., Nomoto et al. 2007). All other recent revisions relevant for low- and intermediate-mass binary evolution in the code are described in detail in Belczynski et al. (2008). Additionally, in the Belczynski et al. (2005) study, very different criteria were adopted for SNe la; the majority of their supernovae originated from sub-Chandrasekhar mass events and it was permitted that the merger of two WDs of any type (including ONeMg WDs) with a total mass exceeding $1.4 \mathrm{M}_{\odot}$ led to a SN Ia. In the current study we also note that the number of sub-Chandrasekhar mass events exceed the number of Chandrasekhar mass progenitors. The weakness of the sub-Chandrasekhar model is that much of the outer (and fastest moving) material is believed to burn to nickel with very few intermediate mass elements (Livne \& Arnett 1995). The resulting spectra do not match current observations of normal supernovae, and the best fits are for sub-luminous supernovae. Only $\sim 6$ sub-luminous SNe Ia were recently reported (Kasliwal et al. 2008) as compared with 36 (Riess et al. 1998) or 42 (Strolger et al. 2004) normal ones discovered only in the Hubble surveys.

\section{DISCUSSION}

We have evolved single and binary stars using the population synthesis code StarTrack, and have analyzed the resulting delay times (time from binary formation at $t=0$ to $\mathrm{SN}$ Ia) of potential Type Ia supernovae. We have considered possible $\mathrm{SNe}$ Ia progenitors arising from three formation channels: Double Degenerate Scenario (white dwarf mergers), Single Degenerate Scenario (hydrogen-rich accretion on to a WD) and the AM Canum Venaticorum scenario (helium-rich accretion on to a WD). Additionally, we have computed SN Ia rates for two galaxy types: an elliptical galaxy with a starburst at $t=0$ and a spiral galaxy with a constant SFR, and in each case we have tested the impact of the common envelope removal efficiency for two different parameterizations: $\alpha_{\mathrm{CE}} \times \lambda=1$ (Model 1), and $\alpha_{\mathrm{CE}} \times \lambda=0.5$ (Model 2).

Our SN Ia rates (century) $)^{-1}\left(10^{10} \mathrm{M}_{\odot}\right)^{-1}$ have been presented in Tables 1 and 2 . We reiterate that the rates which we have derived in this work are 'local' (no redshift evolution) rates. It is still interesting to note that in calculating the volumetric SN Ia rate out to $z=0.12$ fitting the A + B model, Dilday et al. (2008) found A $=(2.8 \pm 1.2) \times 10^{-14} \mathrm{SNe} \mathrm{Ia} \mathrm{yr}^{-1} \mathrm{M}_{\odot}^{-1}$, which is most similarly matched by our DDS Model 1 rates for Milky Way-like spirals $\left(\sim 3 \times 10^{-14} \mathrm{SNe} \mathrm{Ia} \mathrm{y^{-1 }} \mathrm{M}_{\odot}^{-1}\right.$ at 10 Gyr). This 2-component or "A+B" model (see Mannucci et al. 2006, Scannapieco \& Bildsten 2005) assumes that the SN Ia rate is a function of stellar mass density and the SFR, and allows for fitting prompt and tardy SN Ia populations. We note that for Model 1, DDS progenitors

\footnotetext{
7 Delay times were computed by adopting a cosmic star forma-
} tion history based on that of Madau et al. (1998). 
dominate the overall SN Ia rates, whether it is for elliptical or spiral hosts. For Model 2, the overall rates of SNe Ia are a factor of two lower than in Model 1, though $\mathrm{SNe}$ Ia progenitors arising from the DDS and SDS channels are found in equal numbers for delay times $2.5-5.5 \mathrm{Gyr}$.

For all models considered, we expect both very short $(\lesssim 1 \mathrm{Gyr})$ and long $(\sim 10-15 \mathrm{Gyr})$ SN Ia delay times if they originate from DDS progenitors. Could such a population explain the bimodal distribution of delay times derived from some observations? In principle, one may expect such a result; if DDS progenitors are dominant in both old and young galaxies, the empirically-derived delay times may appear to be bimodal and erroneously point toward two different progenitor populations. However, the reported bimodal DTD among SNe Ia (Mannucci et al. 2006, i.e., $50 \%$ of SNe Ia having 'prompt' delay times $\lesssim 100 \mathrm{Myr}$ ) is not reproduced in any of our models. For our standard model, only $\sim 5 \%$ of systems have delay times below $100 \mathrm{Myr}$, with $50 \%$ of our $\mathrm{SNe}$ Ia occurring within $\sim 800 \mathrm{Myr}$ since the starburst. The model which comes closest to reproducing such a bimodal delay time distribution is the DDS channel of Model 2, where there is a large fraction of SNe Ia progenitors with short delay times (50\% with $t<500 \mathrm{Myr}$ ). If in fact such a large fraction of SNe Ia occur within $100 \mathrm{Myr}$ of star formation, it may pose a very interesting problem for binary evolution to explain. Some alternatives are already being considered, for example a single star SN Ia progenitor (Iben \& Tutukov 1984; Maoz 2008). To properly approach this issue one needs to fold our evolutionary calculations with the cosmic star formation history and distribution of galaxy types and mass as a function of redshift. However, it was pointed out that the constraints on observational DTDs which incorporate convolution with assumed cosmic star formation history may not be as strong as they are claimed to be (Förster et al. 2006).

For our standard (Model 1) set of evolutionary parameters, the DDS dominates (roughly 90\%) the rate of Type Ia SNe in both spiral and elliptical galaxies. The rate of DDS SNe Ia is consistent with the observed SN Ia rate of Cappellaro et al. (1999). The DTD of potential DDS SNe la follows a smooth power law distribution through a Hubble time. The SDS DTD is mostly flat throughout a Hubble time, while the AM CVn SNe contribute events mostly at short delay times, but neither contribute significantly to the total SN Ia rate for spiral-like galaxies. The DDS DTD, following a power law, is consistent with the findings of Totani et al. (2008), whose observationallyderived delay time of $\mathrm{SNe}$ Ia in intermediate-redshift elliptical galaxies follows a featureless power law $\propto t^{-1}$ for $0.1<t<10$ Gyr.

For Model 2, the DDS dominates (83\%) the rate of Type Ia SNe in both spiral and elliptical galaxies. The DDS rate is below the observed SN Ia rate of Cappellaro et al. (1999) by a factor of two. The DTD of potential DDS SNe la does not exhibit as strong of a power-law shape as Model 1, since relatively more white dwarf binaries merge at early delay times, and more would-be DDS progenitors merge during the $\mathrm{CE}$ phase and thus never produce a SN Ia. The SDS DTD is most prominent $\sim 2-6$ Gyr (at which times the SDS rates match those of the DDS) and at later times the SDS contributes very little to the overall rates. The AM CVn SNe con- tribute some events mostly at short delay times, with the rates being at a very low level at long delay times.

As noted previously, all DDS SNe Ia progenitors are the result of a merger of a CO-CO WD binary. Mergers between $\mathrm{CO}-\mathrm{He}$ WDs are relatively less common than $\mathrm{He}-\mathrm{He}$ or CO-CO WD mergers since often times mass transfer is stable, and upon reaching contact the CO-He binary will enter an AM CVn phase rather than coalesce. In any case, mergers of $\mathrm{He}-\mathrm{He}$ or $\mathrm{CO}-\mathrm{He}$ WDs are not massive enough to lead to SNe Ia, though they may lead to other very interesting phenomena, as they are likely precursors to helium-burning hot subdwarfs or $\mathrm{R} \mathrm{CrB}$ stars (Webbink 1984). There is a marked decrease in the number of double white dwarfs which are formed in Model 2 relative to Model 1 due to a heightened number of mergers which are encountered during the CE phase.

Typically, population synthesis calculations produce the right number of Chandrasekhar mass WD-WD mergers, yet usually produce an order of magnitude too few SDS supernovae to explain the observed rate estimates (see Livio 2001, for a review). As it turns out, most hydrodynamical white dwarf merger calculations result in the less massive white dwarf being disrupted in a few orbits, causing the more massive white dwarf to accrete at an extremely high rate (Rasio \& Shapiro 1995; Benz et al. 1989). At very high accretion rates, the white dwart mergers are believed to collapse to form a neutron star, not produce a SN Ia thermonuclear explosion \begin{tabular}{l|l|l|l|}
\hline Mochkovitch \& Livio 1990, Saio \& Nomoto 1985, 1998 \\
\hline
\end{tabular} Woosley \& Weaver 1994). Some new studies have found that some mergers might produce Type Ia supernovae, but the merger conditions must be severely refined (Yoon et al. 2007).

Either population synthesis studies with the adopted accretion physics are missing active progenitor paths for Type Ia supernovae, or the physics of merger calculations are incorrect. All of our results and conclusions were based on one evolutionary model with a specific (albeit the most updated) set of accumulation rates and have been presented from the standpoint of population synthesis (i.e., thus far we have ignored the information provided by merger calculations). Yoon et al. (2007) predicted that only DDS systems with small mass ratios $q<0.4$ can produce SN Ia explosions, while the rest will end up in accretion induced collapse and neutron star formation. If we had adopted this as an additional criterion the implications would have been rather dramatic for our results. At the time of the merger, only $\sim 0.2 \%$ $(0 \%)$ of the DDS systems from Model 1 (Model 2) have $q<0.4$, since a low $q$ usually leads to stable mass transfer and not a merger. If the DDS rates had been decreased so drastically, none of our calculated SN Ia progenitors would be able to match the observed rates.

If we had relaxed our assumption on Chandrasekhar mass explosions and we had incorporated subChandrasekhar mass explosion models - ignition of a degenerate layer of He-rich material accumulated on the surface of a WD ${ }^{8}$ Woosley \& Weaver 1994, Kato \& Hachisu 1999) - the Galactic rates for AM CVn would increase from $\lesssim 10^{-4} \mathrm{yr}^{-1}$ to $\sim 10^{-3} \mathrm{yr}^{-1}$ for Model 1 and to $\sim 5 \times 10^{-\widetilde{4}}$ for Model 2. We note that in this estimate

\footnotetext{
8 The sub-Chandrasekhar mass model falls under our AM CVn formation channel.
} 
we have allowed sub-Chandrasekhar mass explosions to occur only for white dwarfs with masses $M_{\mathrm{wd}}>1 \mathrm{M}_{\odot}$, since for lower WD masses the explosions would not look like those observed for typical Type Ia supernovae (e.g., Hillebrandt \& Niemeyer 2000).

If indeed white dwarf mergers (DDS) cannot lead to thermonuclear explosions, one needs to consider alternatives for increasing the rates of SDS and AM CVn progenitors, either by moving away from the standard evolutionary model, or widening the range for efficient accumulation rates onto white dwarfs.
AJR acknowledges a New Mexico State University Graduate Research Enhancement Grant and a Meritbased Enhancement Award, as well the Supernova Rates 2008 LOC for support. KB acknowledges the support from KBN grant N N203 302835. AJR and KB are thankful to L.Bildsten, K.Shen, R.Taam, L.Strolger, P.Podsiadlowski, K.Nomoto and T.Totani for informative discussion. AJR is additionally grateful to J.Grindlay for stimulating discussion, to J.Holtzman for useful suggestions while preparing this manuscript, and thanks B.Dilday and G.Nelemans for helpful information.

\section{REFERENCES}

Abt, H. A. 1983, ARA\&A, 21, 343

Applegate, J. H. 1991, ApJ, 370, 324

Belczynski, K., Kalogera, V., Rasio, F. A., Taam, R. E., Zezas, A., Bulik, T., Maccarone, T. J., \& Ivanova, N. 2008, ApJS, 174, 223

Belczynski, K., Bulik, T., \& Ruiter, A. J. 2005, ApJ, 629, 915 Belczynski, K., Kalogera, V., \& Bulik, T. 2002, ApJ, 572, 407

Benz, W., Thielemann, F.K., \& Hills, J.G. 1989, ApJ, 342, 986

Branch, D., \& van den Bergh, S. 1993, AJ, 105, 2231

Calura, F., \& Matteucci, F. 2006, ApJ, 652, 889

Cappellaro, E., Evans, R., \& Turatto, M. 1999, A\&A, 351, 459

Cox, A. N. (Editor) 2000, Allen's Astrophysical Quantities,

Fourth Ed., Springer-Verlag, New York, NY

Dahlen, T., Strolger, L.-G., \& Riess, A. G. 2008, ApJ, 681, 462

de Donder, E., \& Vanbeveren, D. 2003, New Astronomy, 8, 817

Della Valle, M., Panagia, N., Padovani, P., Cappellaro, E.,

Mannucci, F., \& Turatto, M. 2005, ApJ, 629, 750

Della Valle, M., \& Panagia, N. 2003, ApJ, 587, L71

Dilday, B., et al. 2008, ApJ, 682, 262

Duquennoy, A., \& Mayor, M. 1991, A\&A, 248, 485

Fisher, J., Schröder, K.-P., \& Smith, R. C. 2005, MNRAS, 361, 495

Förster, F., Wolf, C., Podsiadlowski, P., \& Han, Z. 2006,

MNRAS, 368, 1893

Greggio, L. 2005, A\&A, 441, 1055

Hachisu, I., Kato, M., \& Nomoto, K. 2008, ApJ, 683, L127

Hachisu, I., Kato, M., \& Nomoto, K. 1999, ApJ, 522, 487

Hamuy, M., Phillips, M. M., Maza, J., Suntzeff, N. B., Schommer, R. A., \& Aviles, R. 1995, AJ, 109, 1

Han, Z., \& Podsiadlowski, P. 2006, MNRAS, 368, 1095

Han, Z., \& Podsiadlowski, P. 2004, MNRAS, 350, 1301

Hashimoto, M.-A., Nomoto, K.-I., Arai, K., \& Kaminisi, K. 1986, ApJ, 307, 687

Hillebrandt, W., \& Niemeyer, J. C. 2000, ARA\&A, 38, 191

Hurley, J. R., Pols, O. R., \& Tout, C. A. 2000, MNRAS, 315, 543

Iben, I., Jr., \& Renzini, A. 1983, ARA\&A, 21, 271

Iben, I., Jr., \& Tutukov, A. V. 1984, ApJS, 54, 335

Ivanova, N., \& Taam, R. E. 2003, ApJ, 599, 516

Kasliwal, M. M., et al. 2008, ApJ, 683, L29

Kato, M., \& Hachisu, I. 1999, ApJ, 513, L41

Kato, M., \& Hachisu, I. 2004, ApJ, 613, L129

Klypin, A., Zhao, H., \& Somerville, R. S. 2002, ApJ, 573, 597

Kobulnicky, H. A., \& Fryer, C. L. 2007, ApJ, 670, 747

Kroupa, P., Tout, C. A., \& Gilmore, G. 1993, MNRAS, 262, 545

Lada, C. J. 2006, ApJ, 640, L63

Lépine, S., \& Bongiorno, B. 2007, AJ, 133, 889

Livio, M., \& Riess, A. G. 2003, ApJ, 594, L93

Livio, M. 2000, Type Ia Supernovae, Theory and Cosmology, 33

Livio, M. 2001, Supernovae and gamma-ray bursts: the greatest explosions since the Big Bang. Proceedings of the Space

Telescope Science Institute Symposium, held in Baltimore, MD, USA, May 3 - 6, 1999, edited by Mario Livio, Nino Panagia, Kailash Sahu. Space Telescope Science Institute symposium series, Vol. 13. Cambridge, UK: Cambridge University Press

Livne, E., \& Arnett, D. 1995, ApJ, 389, 695

Lü, G., Yungelson, L., \& Han, Z. 2006, MNRAS, 372, 1389

Madau, P., della Valle, M., \& Panagia, N. 1998, MNRAS, 297, L17

Mannucci, F., Della Valle, M., Panagia, N., Cappellaro, E., Cresci, G., Maiolino, R., Petrosian, A., \& Turatto, M. 2005 A\&A, 433, 807

Mannucci, F., Della Valle, M., \& Panagia, N. 2006, MNRAS, 370, 773
Maoz, D. 2008, MNRAS, 384, 267

Maoz, D., \& Gal-Yam, A. 2004, MNRAS, 347, 951

Matteucci, F., \& Greggio, L. 1986, A\&A, 154, 279

Mazeh, T., Goldberg, D., Duquennoy, A., \& Mayor, M. 1992, ApJ, 401, 265

Miyaji, S., Nomoto, K., Yokoi, K., \& Sugimoto, D. 1980, PASJ, 32,303

Mochkovitch, R., \& Livio, M. 1990, A\&A, 236, 378

Nelemans, G. 2005, The Astrophysics of Cataclysmic Variables and Related Objects, 330, 27

Nelemans, G., \& Tout, C. A. 2005, MNRAS, 356, 753

Nelemans, G., Yungelson, L. R., \& Portegies Zwart, S. F. 2004, MNRAS, 349, 181

Nelemans, G., Yungelson, L. R., \& Portegies Zwart, S. F. 2001, A\&A, 375, 890

Nomoto, K., Saio, H., Kato, M., \& Hachisu, I. 2007, ApJ, 663, 1269

Nomoto, K., Iwamoto, K., \& Kishimoto, N. 1997, Science, 276, 1378

Nomoto, K. 1982, ApJ, 253, 798

O'Shaughnessy, R., Kim, C., Kalogera, V., \& Belczynski, K. 2008, ApJ, 672, 479

Perlmutter, S., et al. 1997, ApJ, 483, 565

Perlmutter, S., et al. 1999, ApJ, 517, 565

Peters, P. C. 1964, Physical Review , 136, 1224

Phillips, M. M. 1993, ApJ, 413, L105

Podsiadlowski, P. 2008, Hydrogen-Deficient Stars , 391, 323

Prialnik, D., \& Kovetz, A. 1995, ApJ, 445, 789

Rasio, F. A., \& Shapiro, S. L. 1995, ApJ, 438, 887

Ricker, P. M., \& Taam, R. E. 2008, ApJ, 672, L41

Riess, A. G., et al. 1998, AJ, 116, 1009

Riess, A. G., Press, W. H., \& Kirshner, R. P. 1995, ApJ, 438, L17

Saio, H., \& Nomoto, K. 1985, A\&A, 150, L21

Saio, H., \& Nomoto, K. 1998, ApJ, 500, 388

Scannapieco, E., \& Bildsten, L. 2005, ApJ, 629, L85

Schmidt, B. P., et al. 1998, ApJ, 507, 46

Solheim, J.-E., \& Yungelson, L. R. 2005, 14th European

Workshop on White Dwarfs, 334, 387

Sparks, W. M., \& Stecher, T. P. 1974, ApJ, 188, 149

Strolger, L.-G., et al. 2004, ApJ, 613, 200

Sullivan, M., et al. 2006, ApJ, 648, 868

Thielemann, F.-K., Brachwitz, F., Höflich, P., Martinez-Pinedo, G., \& Nomoto, K. 2004, New Astronomy Review, 48, 605

Totani, T., Morokuma, T., Oda, T., Doi, M., \& Yasuda, N. 2008, PASJ, 60, 1327

Trimble, V. 1990, MNRAS, 242, 79

Tutukov, A. V., \& Yungelson, L. R. 1979, Acta Astronomica, 29, 665

van der Sluys, M. V., Verbunt, F., \& Pols, O. R. 2006, A\&A, 460, 209

Warner, B. 1995, Ap\&SS, 225, 249

Webbink, R. F. 1984, ApJ, 277, 355

Whelan, J., \& Iben, I. J. 1973, ApJ, 186, 1007

Woosley, S. E., \& Weaver, T. A. 1994, ApJ, 423, 371

Yoon, S.-C., Podsiadlowski, P., \& Rosswog, S. 2007, MNRAS,

380, 933

Yungelson, L. R., \& Livio, M. 2000, ApJ, 528, 108

Yungelson, L., \& Livio, M. 1998, ApJ, 497, 168 
TABLE 1

Rates of SNe Ia Progenitors (SNuM) for Model 1

\begin{tabular}{lcc}
\hline \hline Rate $\left[(100 \mathrm{yr})^{-1} 10^{10} \mathrm{M}_{\odot}^{-1}\right]$ as a Function of Time. & \\
\hline DDS & Elliptical & Spiral \\
$0.5 \mathrm{Gyr}$ & $1.6 \times 10^{-1}$ & $2.0 \times 10^{-1}$ \\
$3 \mathrm{Gyr}$ & $2.3 \times 10^{-2}$ & $8.0 \times 10^{-2}$ \\
$5 \mathrm{Gyr}$ & $1.2 \times 10^{-2}$ & $6.0 \times 10^{-2}$ \\
$10 \mathrm{Gyr}$ & $\sim 5 \times 10^{-3}$ & $3.3 \times 10^{-2}$ \\
SDS & & \\
$0.5 \mathrm{Gyr}$ & $\lesssim 10^{-3}$ & 0 \\
$3 \mathrm{Gyr}$ & $\sim 1 \times 10^{-3}$ & $\approx 10^{-3}$ \\
$5 \mathrm{Gyr}$ & $\lesssim 10^{-3}$ & $\sim 10^{-3}$ \\
$10 \mathrm{Gyr}$ & & $\sim 10^{-2}$ \\
AM CVn & $2.2 \times 10^{-2}$ & $\sim 5 \times 10^{-3}$ \\
$0.5 \mathrm{Gyr}$ & $<10^{-3}$ & $\sim 4 \times 10^{-3}$ \\
$3 \mathrm{Gyr}$ & $\lesssim 10^{-4}$ & $\sim 1 \times 10^{-3}$ \\
$5 \mathrm{Gyr}$ & 0 & \\
\hline $10 \mathrm{Gyr}$ & & \\
\hline
\end{tabular}

TABLE 2

Rates of SNe Ia Progenitors (SNuM) For Model 2

\begin{tabular}{lcc}
\hline \hline Rate $\left[(100 \mathrm{yr})^{-1} 10^{10} \mathrm{M}_{\odot}^{-1}\right]$ as a Function of Time. & \\
\hline DDS & Elliptical & Spiral \\
$0.5 \mathrm{Gyr}$ & $1.3 \times 10^{-1}$ & $1.3 \times 10^{-1}$ \\
$3 \mathrm{Gyr}$ & $\sim 5 \times 10^{-3}$ & $6.0 \times 10^{-2}$ \\
$5 \mathrm{Gyr}$ & $\sim 3 \times 10^{-3}$ & $3.5 \times 10^{-2}$ \\
$10 \mathrm{Gyr}$ & $\sim 10^{-3}$ & $1.4 \times 10^{-2}$ \\
SDS & $\sim 2 \times 10^{-3}$ & 0 \\
$0.5 \mathrm{Gyr}$ & $\sim 5 \times 10^{-3}$ & $\sim 8 \times 10^{-3}$ \\
$3 \mathrm{Gyr}$ & $\sim 3 \times 10^{-3}$ & $\sim 6 \times 10^{-3}$ \\
$5 \mathrm{Gyr}$ & $\sim 10^{-4}$ & $\sim 3 \times 10^{-3}$ \\
$10 \mathrm{Gyr}$ & & $<10^{-3}$ \\
AM CVn & $\sim 5 \times 10^{-3}$ & $\sim 10^{-3}$ \\
$0.5 \mathrm{Gyr}$ & 0 & $\sim 10^{-3}$ \\
$3 \mathrm{Gyr}$ & 0 & $<10^{-3}$ \\
$5 \mathrm{Gyr}$ & $<10^{-4}$ & \\
\hline
\end{tabular}




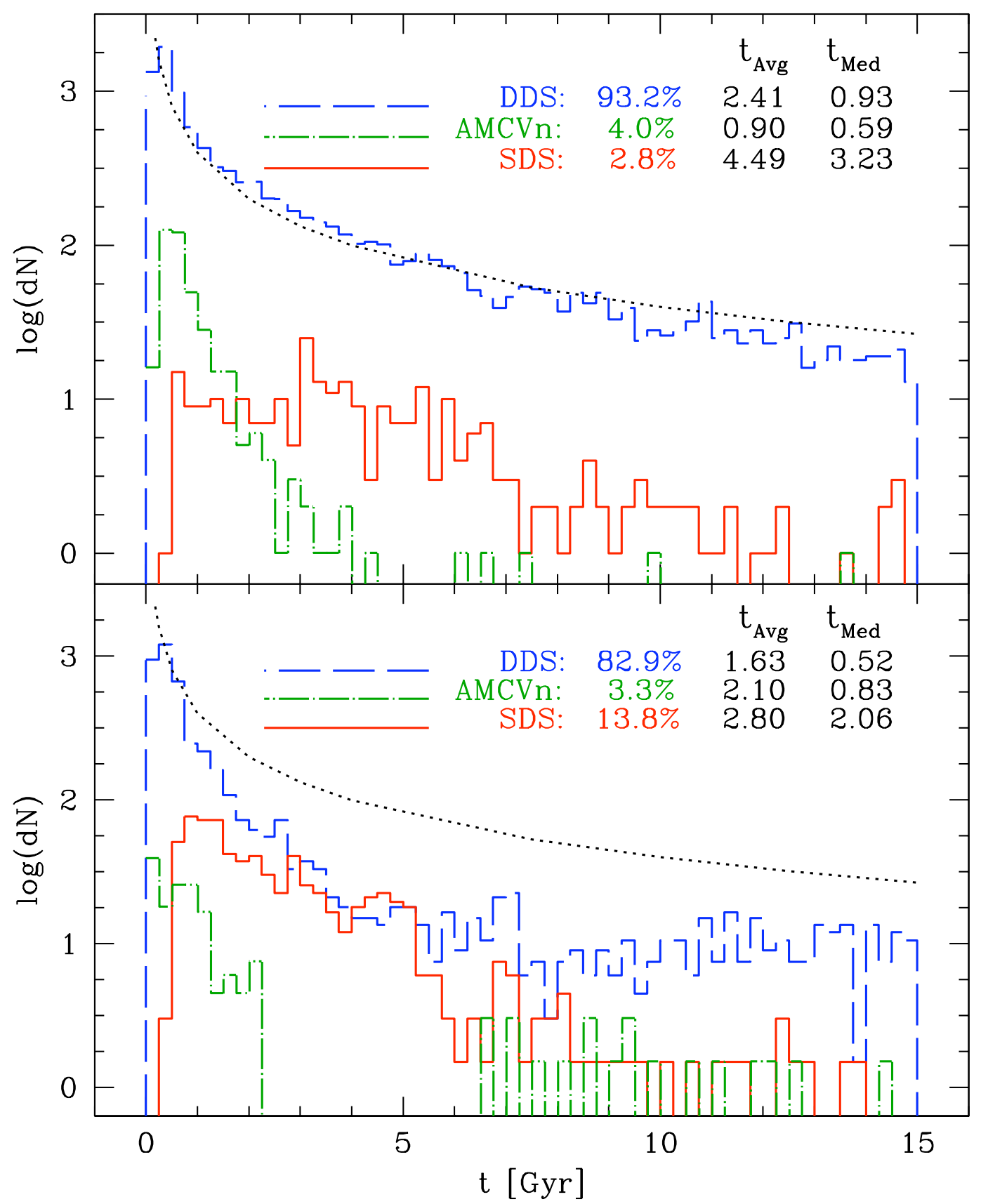

FIG. 1.- Delay time distribution showing relative contributions from the three SN Ia formation channels considered in this work for our elliptical galaxy (instantaneous burst of star formation at $t=0$ ): DDS (blue dashed), AM CVn (green dot-dash) and SDS (red solid). Average and median delay times are indicated. Top panel: Model $1, \alpha_{\mathrm{CE}} \times \lambda=1.0$. The thin dotted line represents a power law function of form $f(t)=100 t^{-1}$ and follows the DDS delay time distribution reasonably well. Bottom panel: Model $2, \alpha_{\mathrm{CE}} \times \lambda=0.5$. The power law is shown for comparison. 


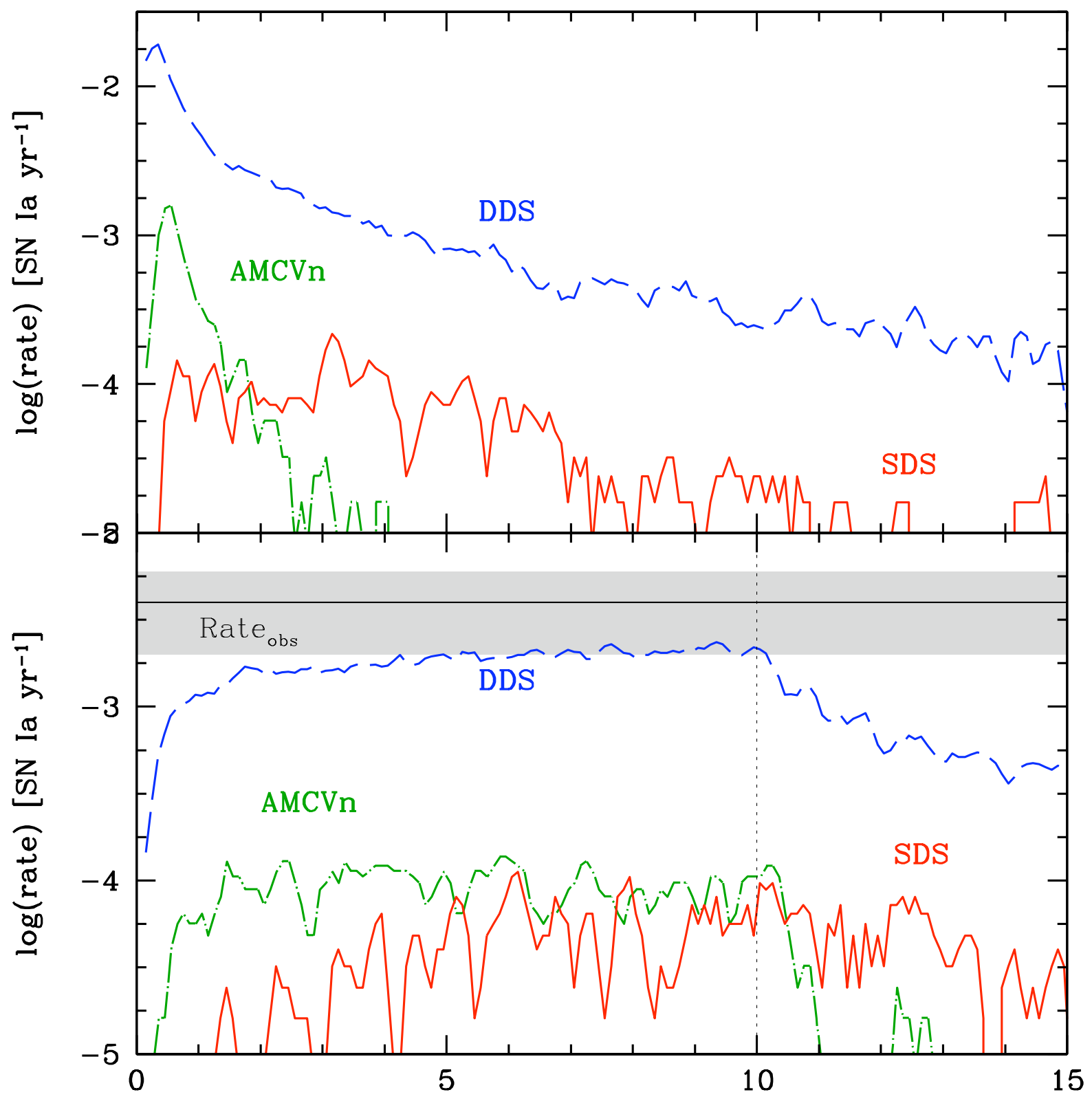

FIG. 2.- Rates of Type Ia supernovae (number per year) for the Model 1 galaxies. The DDS (blue dashed), AM CVn (green dot-dash) and SDS (red solid) channel rates are shown. Top panel: elliptical galaxy whose total mass in formed stars is $\mathrm{M}_{\text {tot }}=6 \times 10^{10} \mathrm{M}_{\odot}$ (delta function starburst at $t=0$ ). Bottom panel: spiral (constant star formation) galaxy whose total mass in formed stars at 10 Gyr is $\mathrm{M}_{\mathrm{tot}}=6 \times 10^{10} \mathrm{M}_{\odot}$. Approximating the star formation history of the MW to be constant, the current Galactic SN Ia rate (shown with vertical dotted line) considering the DDS alone is $0.002 \mathrm{yr}^{-1}$. This is consistent with the empirical rate as indicated by the shaded region in the plot $\left(0.004 \pm 0.002 \mathrm{yr}^{-1}\right.$, Cappellaro et al. 1999). 


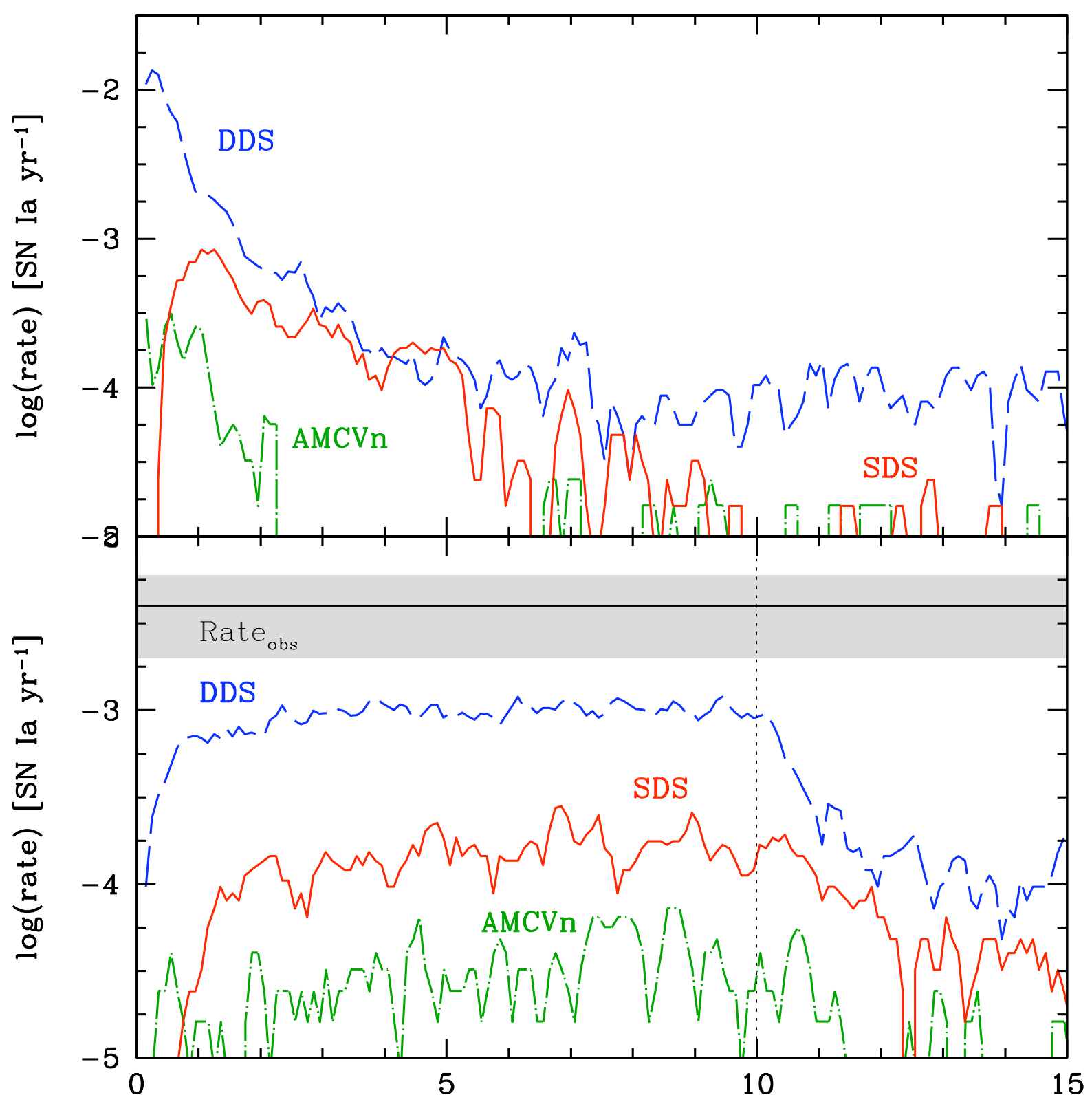

Fig. 3.- Same as Figure 2 for the Model 2 stellar population. Top panel: elliptical galaxy. Bottom panel: spiral galaxy. Approximating the star formation history of the MW to be constant, the current Galactic SN Ia rate considering the DDS alone is $0.0009 \mathrm{yr}^{-1}$. The combined (DDS + SDS + AM CVn) rate is $0.001 \mathrm{yr}^{-1}$, which is below the empirical rate estimate of Cappellaro et al. (1999; $0.004 \pm 0.002$ $\left.\mathrm{yr}^{-1}\right)$. 